\title{
Latent place learning in a novel environment and the influences of prior training in rats
}

\author{
JULIAN R. KEITH and KATHERINE M. MCVETY \\ University of Colorado, Boulder, Colorado
}

\begin{abstract}
Latent place learning in a novel environment was studied in rats, using a variation on the Morris (1981) water task. Groups of rats received 30 trials over 3 days in a training environment in which they learned to swim to an escape platform, were permitted to swim around the maze with no platform, or were placed on the platform by the experimenter. In a subsequent test, groups that had learned to swim to the escape platform during initial training were quicker to locate the platform in a novel environment after viewing the room from the location of the escape platform than were similarly pretrained subjects that were not allowed to view the room from the platform. Groups that were not trained to swim to the platform but received training on other procedural aspects of the task in the first environment did not benefit from standing on the escape platform in the novel environment. These results show that animals that are familiar with the requirements of the water maze task can generate accurate trajectories through a novel area based on information obtained while viewing the environment from the goal location.
\end{abstract}

The degree to which knowledge can be acquired independently of the behavior of the organism is a longstanding and controversial issue in learning theory (see, e.g., Tolman, 1948). Spatial mapping theory, in particular, proposes that knowledge about the spatial relationships between objects in an environment is represented in a form that is independent of behavior (Morris, 1981; O'Keefe $\&$ Nadel, 1978). Some of the most promising recent approaches to this question have used the Morris (1981) place learning task. Briefly, the original version of the task employed by Morris required rats to swim to an escape platform that was slightly submerged in a circular swimming pool. Although the rats were unable to see the platform itself, they readily learned to navigate to it from a variety of starting locations, presumably by learning the location of the platform in relation to extramaze cues.

One of the more provocative findings of Morris's (1981) original study was the observation that well-trained rats swam directly to the hidden platform even when they were started from a novel location. Morris referred to this capacity as "instantaneous transfer," implying that the computations that permit an animal to generate accurate trajectories through a novel space are based on knowledge acquired from different views of that environment. These findings have been interpreted as strong evidence for the presence in rats of cognitive mapping abilities, as described in the classic literature of animal learning (e.g., Tolman, 1948).

The authors thank Jerry Rudy for providing the equipment used in this study and for his helpful discussions during the preparation of this manuscript. We would also like to thank Carol Barnes and Bruce McNaughton for providing financial support for this experiment and for their comments, and E. J. Green for his help and comments. Address correspondence to Julian R. Keith, Department of Psychology, Campus Box 345, University of Colorado, Boulder, CO 80309.
Further evidence that the rat's knowledge of its spatial environment is stored independently of its behavior was provided by Sutherland and Linggard (1982), who employed a latent learning paradigm (Tolman, 1948). Naive rats were placed on the escape platform and simply allowed to view the distal cue environment. Compared with rats that did not receive this training, the rats with the latent learning experience learned more rapidly to navigate to the platform, suggesting that they learned something about the location of the platform while standing on it. The effect, however, was not large.

More recently, however, Sutherland, Chew, Baker, and Linggard (1987) reported data that challenge the view that the rat can learn the location of a goal without actually engaging in goal searching behavior. They trained rats to navigate to an escape platform from a variety of starting locations. However, during training some of the animals were prevented by a Plexiglas barrier from visiting one portion of the pool or viewing the room from that region. Later, when tested from starting positions on the side of the pool that was previously restricted, they navigated poorly. Other rats, which received the same training but were allowed to swim through the restricted area or view the room from there, were able to locate the escape platform from starting positions in all portions of the pool with shorter latencies.

Sutherland et al. (1987) interpreted these results as suggesting that rats need to have prior experience in moving through or viewing an environment from a given location in order to navigate directly from that location to their goal. This evidence, in their view, weighs heavily against the notion of instantaneous transfer in rats. Indeed, Sutherland et al. (1987) attributed the previous finding of Sutherland and Linggard (1982) to the fact that Sutherland and Linggard allowed the rats in their study to swim around 
the pool before ever placing the animals on the platform. They also noted that the training procedures used by Morris (1981) allowed the animals to swim through much of the pool, including the "novel" starting position used on the test trial, during the early phases of training.

Taken at face value, the findings of Sutherland et al. (1987) could be viewed as evidence against both the notion that the rat can learn locations without ever actively exploring the environment and the notion that the knowledge of place locations is independent of the behavioral actions used to locate goals. It is possible, however, that the training procedures used by Sutherland and Linggard (1982) and Sutherland et al. (1987) obscured the very capacities that they were intended to measure. In the former study, the subjects did not have an opportunity to master the procedural (nonspatial) requirements of the task, such as swimming to and climbing onto the escape platform, before being tested. Because the subject needs to have mastered the procedural aspects of the task in order to demonstrate place learning in the water maze, Sutherland and Linggard (1982) may have grossly underestimated the rat's capacity for this type of learning.

In the present study, we reexamined the latent placelearning capacities of the rat. The subjects were pretrained on some of the procedural components of the task in one environment before latent place learning was examined in a novel environment. The results indicate that rats that have been trained to locate a hidden platform in one environment can learn the location of a hidden platform in a new environment while viewing the room from the platform.

\section{METHOD}

\section{Subjects}

Forty-nine Long-Evans hooded rats were used in this study. All subjects were between 60 and 90 days of age. Sex was undetermined in the present experiment, because we had been unable to detect differences between sexes on the Morris water task in numerous pilot studies. All animals were obtained from the breeding facilities at the University of Colorado. The subjects were housed in large tub cages in groups of 6 and had continuous access to Purina rat chow and water. The colony was on a 12:12-h light:dark cycle, and all behavioral training and testing were done during the dark phase of the cycle.

\section{Experimental Design}

There were seven groups in the present experiment. During Phase 1 (training), the subjects in four of these groups (View-View [V-V], View-180 [V-180], View-No View [V-NV], and No ViewView [NV-V]) were trained to locate a hidden platform. (Note that the first half of each abbreviated group name refers to the Phase 1 condition and the second half refers to the Phase 2 condition. Figure 1 and Table 1 provide a summary and description of the Phase 1 and Phase 2 conditions for each group.) Subjects in three of these groups (V-V, V-180, V-NV) were always placed on the platform for $30 \mathrm{sec}$ prior to each training trial. Subjects in group NV-V were released into the pool during training without viewing the room from the platform prior to the trial.

During Phase 2 (testing in a new room), Groups V-V, V-180, and $N V-V$ viewed the novel environment from the escape platform prior to being placed in the water. For Group V-180, immediately

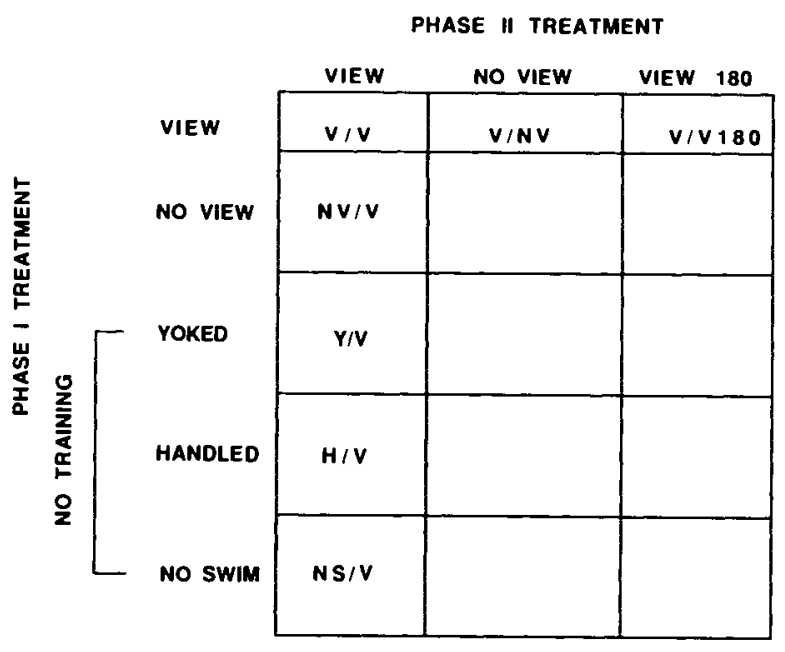

Figure 1. Summary of conditions.

prior to the search period, the platform was placed in the $180^{\circ} \mathrm{di}$ agonally opposite quadrant from where it had been during the viewing period. If viewing the room from the location of the escape platform biases the subject's tendency to return to that location, animals in Group V-180 should take longer to find the platform on the test trial than those in Group V-V. Group NV-V was included to determine whether it was necessary for animals to learn during Phase 1 training that the distal cue information available to them while standing on the platform was relevant to learning the location of the platform. We predicted that if this was the case, Group NV-V would not locate the platform in the novel environment as readily as Group $\mathrm{V}-\mathrm{V}$, since they had not received training viewing the platform prior to trials in Phase 1. Group V-NV was included to assess how readily well-trained subjects could locate the hidden platform in a novel environment without having viewed the new environment from the location of the platform.

Table 1

Phase 1 and Phase 2 Conditions

\begin{tabular}{|c|c|c|}
\hline Group & Phase 1 & Phase 2 \\
\hline $\begin{array}{l}\text { View-View } \\
\text { (V-V) }\end{array}$ & $\begin{array}{l}\text { Viewed room, located } \\
\text { platform by swimming }\end{array}$ & $\begin{array}{l}\text { Viewed room } \\
\text { from platform }\end{array}$ \\
\hline $\begin{array}{l}\text { No View-View } \\
\text { (NV-V) }\end{array}$ & $\begin{array}{l}\text { Located platform } \\
\text { by swimming }\end{array}$ & $\begin{array}{l}\text { Viewed room } \\
\text { from platform }\end{array}$ \\
\hline
\end{tabular}

View-180

(V-180)

Viewed room, located platform by swimming

View-No View Viewed room, located (V-NV) platform by swimming

Yoked-View (Y-V)

\section{Swam for the same} amount of time on each trial as Group V-V, but were not perminted to locate platform

Handled-View Were handled and placed (H-V)

Were handled and placed
in water, did not swim or stand on platform

No Swim-View Viewed room from plat(NS-V) form, were not permitted to swim or locate platform
Viewed room from platform in $180^{\circ}$ opposite quadrant

Did not view room from platform

Viewed room from platform

Viewed room from platform

Viewed room from platform 
The training given to three other groups (Yoked-View [Y-V], No Swim-View [NS-V], and Handled-View [H-V]) did not allow the animals to learn to locate the escape platform during Phase 1, but provided control comparisons for various procedural aspects of the task, such as swimming $(\mathrm{Y}-\mathrm{V})$, viewing the training environment from the platform (NS-V), and being handled by the experimenter $(\mathrm{H}-\mathrm{V})$. During Phase 2 these animals all received treatment identical to that of Group V-V; that is, during Phase 2 they viewed the environment from the platform prior to the search period.

\section{Apparatus}

Three circular water mazes with identical dimensions $(1.2 \mathrm{~m}$ in diameter, $36 \mathrm{~cm}$ deep) were used. The interiors of the two pools used during training were painted gray. The interior of the pool used for testing was painted white. A clear circular Plexiglas escape platform, $11.5 \mathrm{~cm}$ in diameter, was submerged $1 \mathrm{~cm}$ below the surface of the water. The water was clouded by dissolving nontoxic Crayola white paint powder in the pools prior to a session. The water temperature was maintained at $26^{\circ} \mathrm{C}\left( \pm 2^{\circ}\right)$.

The rooms that housed the mazes had identical dimensions $(2.3$ $\times 2.73 \times 2.5 \mathrm{~m}$ ), and differed primarily with respect to the locations of objects that were mounted on the walls and the particular objects used. Each training room had bare walls that were painted off-white; a print of either Einstein or an ancient Japanese Go player hung on the north wall. The west wall of each room held a small cardboard box. Lighting was provided by two $60-\mathrm{W}$ incandescent bulbs mounted in the ceiling over the north and south boundaries of the mazes. All of the mazes were positioned in the center of the rooms that contained them.

The room used for testing had three walls that were covered by white curtains. Illumination was provided by $100-\mathrm{W}$ fluorescent lights mounted at the tops of the north, east, and west walls. A poster of Humphrey Bogart hung on the north wall, a small box was mounted on the west wall, and an artificial window $(43 \times 56 \mathrm{~cm})$ hung on the east wall. An equipment rack that held a video monitor, a VCR, and a computer was located in the southwest corner of the room. A camera mounted directly over the center of the maze permitted the behavior of the subjects to be observed and recorded on videotape for later analysis.

\section{Procedure}

Phase 1: Training. Training took place on 3 consecutive days (10 trials per day). For each particular animal, all training took place in the same room each day. Three of the groups received identical training. The subjects in Groups V-V $(n=8), \mathrm{V}-180(n=8)$, and V-NV $(n=8)$ were placed on the platform for $30 \mathrm{sec}$ (the viewing period) prior to being released from a randomly selected area at the boundary of the maze. During a given session (10 trials), the platform remained in the same location; each day, however, the platform was moved to a new position prior to that day's training. The subjects in Group NV-V $(n=8)$ were not placed on the platform prior to training trials; in all other respects, their training was identical to that of the three groups described above.

The subjects in Group Y-V $(n=6)$ received trials in which they were allowed to swim in the training tank for the same amount of time for given trials as their matched counterparts in Group V-V; however, the platform was removed from the pool during their training. The subjects in Group NS-V $(n=5)$ were placed on the platform for $30 \mathrm{sec}$ but were never permitted to swim during training. The subjects in Group H-V $(n=6)$ were merely placed in the water on each trial and were immediately removed and returned to their home cages.

During the posttrial period, the subjects were allowed to stand on the platform for $5 \mathrm{sec}$ before being returned to their home cages. The intertrial interval was approximately $5 \mathrm{~min}$.

Phase 2: Testing in a novel environment. Twenty-four hours after the third day of training, the subjects were brought into the new environment. The home cage containing the animals was placed beneath the table that supported the water maze, so that the animals could not obtain a view of the extramaze cues that were visible from within the maze.

The subjects in Groups V-V, NV-V, Y-V, NS-V, and H-V received identical treatment in the test situation. These animals were placed on the hidden escape platform for $2 \mathrm{~min}$ prior to being placed in the water.

The platform was positioned such that its center was always within 6 to $8 \mathrm{~cm}$ from the wall of the maze. For a given animal the platform could be located in the north, south, east, or west quadrant. The subjects were always released into the pool facing the wall of the maze. When the platform was located in the north or south quadrant, the subjects were released in the east or west location, and vice versa. These conditions were counterbalanced across groups.

The subjects in Group V-180 received similar experience. Before they were placed into the water, however, the platform was quickly moved to the quadrant that was $180^{\circ}$ diagonally opposite from where they had stood during the 120 -sec viewing period.

Finally, the subjects in Group V-NV were released into the pool with no opportunity to view the distal cues from the escape platform.

\section{Dependent Variables}

During Phase 1 , the only dependent variable was latency to locate the platform. The experimenter timed each trial, using a stopwatch, and recorded the time on a data sheet. If a subject did not locate the platform within $60 \mathrm{sec}$, it was placed on the platform by the experimenter.

In Phase 2, there were two dependent variables. As in Phase 1, latency to locate the platform was measured. Second, whether or not the animal entered the quadrant that contained the platform first was recorded. The experimenter timed the latency for each subject to find and climb onto the platform. If an animal failed to find the platform within $60 \mathrm{sec}$, it was removed from the pool and a time of $60 \mathrm{sec}$ was recorded. Each trial was videotaped by means of the overhead camera and the trial was subsequently scored according to the first new quadrant entered. To do this, a template that divided the pool into four equal quadrants was placed over the video monitor. The template was positioned so that the point where the animal was released was at the center point of the wall of the maze in one quadrant and the platform was in the center of an adjacent quadrant. If the animal entered the quadrant that contained the platform, a score of 1 was given. If the animal entered the quadrant that did not contain the platform, a score of 0 was given. Each subject received a single test trial.

\section{RESULTS}

\section{Phase 1}

Figure 2 shows the mean latencies to locate the platform on all training trials ( 3 days, 10 trials per day). For simplicity, the latencies of the groups that were allowed to view the environment from the platform prior to the search period (V-V, V-180, V-NV) were collapsed into a single group, which is represented in the figure as Group VIEW. Latencies for Group NV-V, which was not allowed to view the environment from the platform prior to the search period, are also shown in the figure. It is clear from the figure that subjects in both Phase 1 training conditions improved over trials and attained a comparable level of proficiency at locating the escape platform.

It is also noteworthy that on the first trial of Phase 1 training, viewing the room from the platform location 


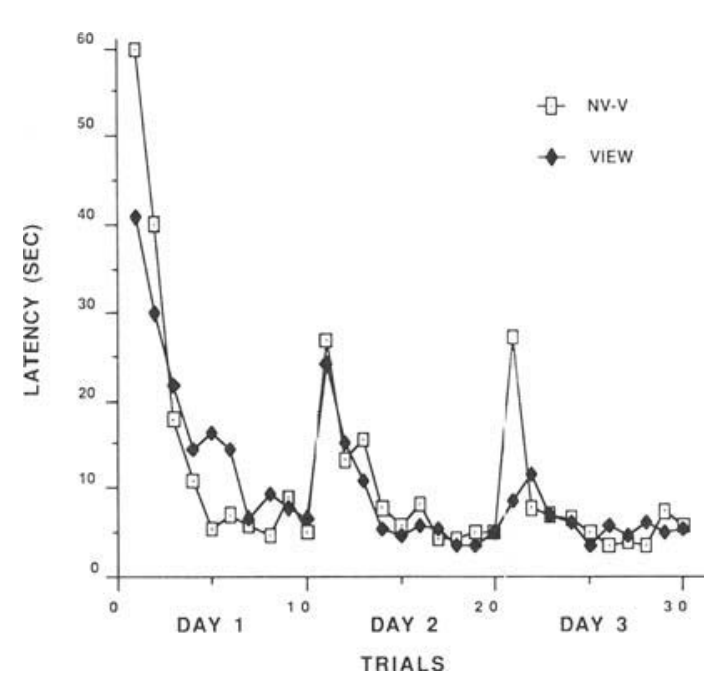

Figure 2. Latencies to locate the escape platform during Phase 1 training for Groups VIEW (consisting of the combined means of groups V-V, V-180, and V-NV and NV-V.

facilitated escape performance. This was confirmed statistically, using a one-tailed $t$ test $[t(14)=3.22, p<.01]$, by combining the means of subjects in Groups $\mathrm{V}-\mathrm{V}$, $\mathrm{V}-180$, and $\mathrm{V}-\mathrm{NV}$ and comparing them with the latencies of subjects in Group NV-V on the first trial of Day 1. It should also be noted that none of the 8 subjects in Group NV-V located the platform on the first trial before the $60-\mathrm{sec}$ cutoff. In contrast, 9 of the 24 subjects that viewed the room from the platform prior to the first trial found the platform.

Recall that during Phase 1 the platform was located in a different area of the pool on each of the 3 training days. As one would expect, latencies to locate the platform were longest on the first trial of each day. By the third day of training, however, the subjects in Group VIEW (Groups $\mathrm{V}-\mathrm{V}, \mathrm{V}-180$, and $\mathrm{V}-\mathrm{NV}$ combined) located the platform with shorter latencies than did those in Group NV-V. This observation was confirmed statistically $[t(14)=2.56$, $p<.05$ ] by combining the means of subjects from Groups V-V, V-180, and V-NV, and comparing them with those of the subjects in Group NV-V on the first trial of the third day.

\section{Phase 2}

Figure 3 shows the mean latencies to locate the escape platform in the new environment. Provided that the animals had learned to locate the escape platform in Phase 1, viewing the novel room from the platform was a sufficient experience to allow them to acquire knowledge about its location. This is demonstrated by the fact that Groups V-V and NV-V had shorter latencies to find the platform than did Groups V-180 and V-NV.

On the other hand, subjects that had been trained on procedural aspects of the task other than swimming to the platform (Groups Y-V, H-V, and NS-V) did not locate the escape platform as quickly as their counterparts that had been trained to swim to the platform (Groups V-V and $\mathrm{NV}-\mathrm{N}$ ), even though they also viewed the novel room from the escape platform prior to the search period.

A one-way analysis of variance (ANOVA) was used to confirm these observations. A significant overall main effect was found between groups $[F(6,42)=3.35$, $p<.01]$. Fisher's $L S D(p=.05)$ revealed that Groups $\mathrm{V}-\mathrm{V}$ and NV-V had significantly shorter latencies than all other groups but did not differ from one another. Additionally, Group V-180 had a significantly shorter average latency than the remaining groups. No other groups differed significantly.

Figure 4 shows the proportion of animals in each group that entered the quadrant containing the escape platform prior to entering any other quadrant on the test trial. A chi-square goodness-of-fit test showed that the observed distribution deviated significantly from the distribution predicted by the hypothesis that viewing the environment from the platform prior to testing would not influence firstquadrant selection $\left[x^{2}(6)=19.1, p<.01\right]$.

Although this test does not reveal which component of the data departs from the normal distribution, close examination of Figure 4 suggests that the bulk of the variability is accounted for by the tendency for animals in Groups V-V and NV-V to select the quadrant that contained the escape platform first. Indeed, 13 of 16 of these animals selected the correct quadrant first $(p=.0085)$.

In order to determine whether escape latencies during Phase 2 for Groups $\mathrm{Y}-\mathrm{V}, \mathrm{H}-\mathrm{V}$, and NS-V were facilitated by the training they received in Phase 1, the latencies of these groups were compared with the combined means of subjects in Groups V-V, V-180, and V-NV on their first trial during Phase 1. The performance of these subjects on the first training trial in Phase 1 is representative of untrained animals that have been allowed

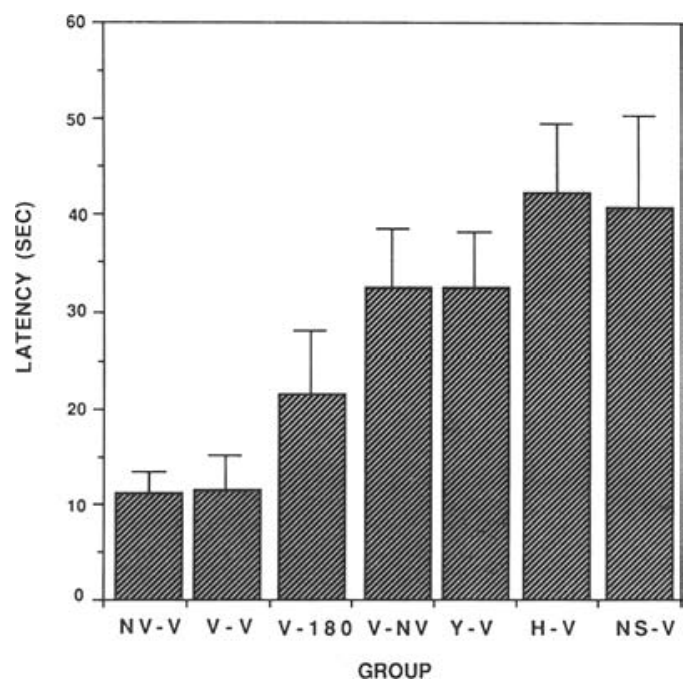

Figure 3. Latencies to locate the escape platform on the test trial in the novel environment for all groups. 


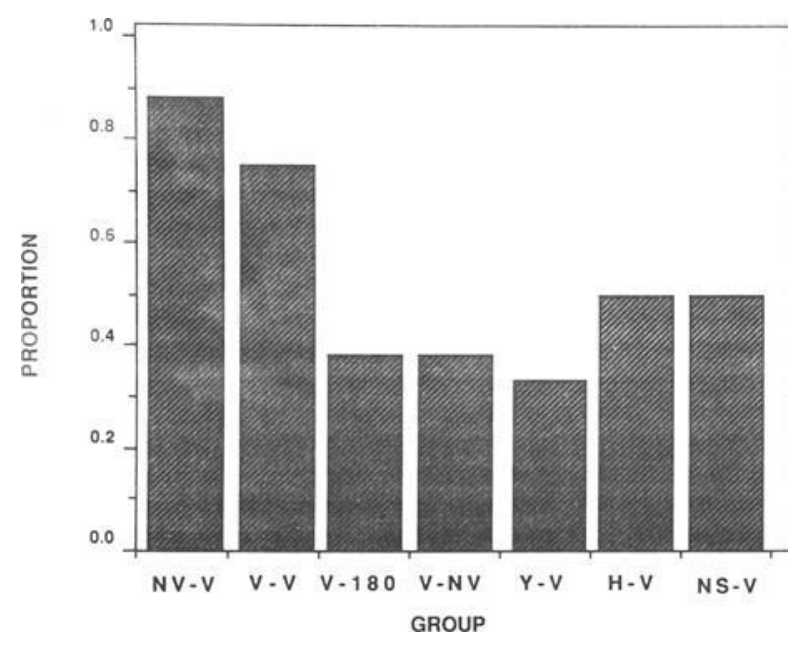

Figure 4. Proportion of animals selecting the quadrant that contained the escape platform first.

to view the room from the platform prior to the search period. No significant differences were found, using a one-way ANOVA $[F(3,21)=1.6, p>.05]$. The escape latencies of subjects in Group NV-V, which were not allowed to view the environment from the platform prior to the search period, on their first trial in Phase 1 were also compared with the latencies of subjects in Groups $\mathrm{Y}-\mathrm{V}, \mathrm{H}-\mathrm{V}$, and NS-V. A significant difference was detected, using a one-way ANOVA $[F(3,21)=3.69$, $p<.05$ ]. Fisher's $L S D$ revealed that only Groups NV-V and $\mathrm{Y}-\mathrm{V}$ differed significantly from one another.

\section{DISCUSSION}

Well-trained rats learned the location of the escape platform in a novel environment simply by viewing the new environment while standing on the platform. This finding strongly suggests that the knowledge system that subserves place learning is independent of the behavioral actions the animal must initiate to navigate to the platform. A comparison of the groups that demonstrated latent learning in this task with those that did not points out important aspects of the task that facilitate the expression of this learning.

The expression of latent place learning is greatly facilitated by prior training in which the animal learns to locate the escape platform by swimming to it. Subjects that were only handled, that were allowed to swim in the pool without locating the platform, or that were allowed to view the room from the platform during Phase 1 located the escape platform in Phase 2 with approximately the same latencies as naive animals that had only been allowed to stand on the platform immediately prior to the trial. That is, subjects in Groups V-V, V-180, and V-NV, which were completely naive when they were placed on the platform prior to the first trial of Phase 1, located the escape platform on their first training trial with about the same latencies as subjects in Groups NS-V, $\mathrm{H}-\mathrm{V}$, and $\mathrm{Y}-\mathrm{V}$ did during Phase 2. Thus, training that did not allow subjects to learn to swim to the platform during Phase 1 did not facilitate performance in Phase 2; simply allowing naive animals to view the room from the platform immediately prior to the trial produced comparable performance.

The degree of facilitation produced by viewing the room from the platform prior to the test trial was profoundly affected by previous training. Although none of the 8 animals that did not view the room from the escape platform prior to the first trial in Phase 1 (Group NV-V) found the platform within the 60 -sec time limit, 9 of the 24 animals that viewed the room from the platform prior to the first trial (Groups V-180, V-V, and V-NV) did find the platform. The escape latencies of these animals were, in every case, considerably longer than the latencies of more extensively trained animals (averaging $33 \mathrm{sec}$, vs. less than $10 \mathrm{sec}$, respectively). These results are reminiscent of those of Sutherland and Linggard (1982).

Similarly, in Phase 2, subjects in Group Y-V found the platform with shorter latencies than did subjects in Group NV-V on Trial 1 of Phase 1. However, subjects in Group $\mathrm{Y}-\mathrm{V}$, unlike subjects trained to find the platform by swimming to it (Groups $\mathrm{V}-\mathrm{V}$ and $\mathrm{NV}-\mathrm{V}$ ), showed no bias toward initially searching in the quadrant where they had previously stood on the platform (see Figure 4).

Taken together, the long escape latencies and the absence of a bias toward initiating searching in the quadrant where they had stood on the platform suggest that animals that had not learned to locate the platform by swimming to it did not learn about the precise location of the platform while standing on it. On the other hand, standing on the platform prior to the trial can alter the search behaviors of some subjects in ways that increase the likelihood of coming into contact with the platform. Perhaps the knowledge that an escape platform is available is sufficient to modify searching behavior and facilitate escape performance.

It is intriguing that Groups $\mathrm{H}-\mathrm{V}$ and NS-V did not locate the platform during Phase 2 any faster than the completely naive subjects in Group NV-V did on their first trial during Phase 1. Based on the performance of all of the other groups that were allowed to view the environment from the platform prior to their first search trial, it would have been predicted that Groups H-V and NS-V would also be superior to Group NV-V on their first experience searching for the platform. We cannot account for this finding without suggesting that the subjects in Groups $\mathrm{H}-\mathrm{V}$ and NS-V learned during Phase 1 training that they would always be returned to their home cages after being handled or placed on the platform, and that the information available during this procedure was irrelevant and therefore not attended to.

If animals acquire information concerning the specific location of the platform during the viewing period, the rats in Group V-180 should have taken longer to find the platform, since the platform was relocated after the view- 
ing period. As expected, the subjects in Group V-180 were slower to locate the platform in Phase 2 than were those in Groups V-V and NV-V. However, their latencies were shorter than expected. At least two factors could have contributed to this finding. First, perhaps the brief experience $(120 \mathrm{sec})$ of viewing the room, while informing the animal about the location of the platform, was not sufficient to produce persistent searching in the original platform location. When the animals in Group V-180 failed to find the platform in its previous location, they may have readily abandoned searching there in favor of another strategy. Second, when the platform was not found in its previous location, alternative search strategies may have been facilitated by information unrelated to the distal cue environment (i.e., availability of a platform, distance between the wall of the maze and the platform, etc.).

Latent place learning did not require that the animal stand on the platform prior to each trial during the training phase, however. Simply allowing the animal to swim to the escape platform and remain on it for 3-5 sec during Phase 1 training enabled the rat to demonstrate latent place learning in the new environment.

Our results join those of Sutherland and Linggard (1982) in demonstrating latent place learning in the rat, but they also suggest that Sutherland and Linggard underestimated this capacity by not taking into account the procedural components of the task that must be mastered in order for this capacity to be more fully expressed.

The results of this experiment stand in sharp contrast to some of the results reported by Sutherland et al. (1987). They found that rats were poorer at finding the escape platform when they started in the region of the pool that they had not visited during training. Sutherland et al. took this finding as evidence that

in order to exhibit transfer of efficient place navigation from a novel starting location in this situation, it is necessary during training for the rats to (1) be familiar with the distal cues viewed from the region of the novel starting location, and (2) swim through the vicinity of the novel starting location. (Sutherland et al., 1987, p. 56)

Furthermore, they concluded that in order for rats to use information obtained while standing on the platform, it was necessary for them to have navigated around the entire pool with unrestricted viewing access to the entire environment. In the present experiment, Groups $\mathrm{V}-\mathrm{V}$ and NV-V displayed knowledge about the location of the escape platform after viewing the environment from the location of the escape platform for only $120 \mathrm{sec}$. Thus, it was not necessary for the animals to have had prior experience either swimming through the novel pool or viewing the novel environment from more than one location in order to learn the location of the platform. It appears that Sutherland et al.'s methodology obscured the spatial cognitive capacities of their subjects. The removal of the partition from the pool appears to have interfered with navigation in the pool in general, except in the groups that received their training with the partition $11 \mathrm{~cm}$ above the surface of the water (Groups A and B). Close inspection of the swim paths shown in Figure 3 of Sutherland et al.'s study reveals that on the test trials, most subjects tended to navigate poorly even when they reached the side of the pool from which they had previously learned to locate the platform. The removal of the partition, a salient cue that marked the boundary of the pool during training, appears to have produced a general navigation deficit for reasons other than those proposed by Sutherland et al.

More work is needed to precisely clarify the nature of the information that animals learn while viewing the room from the escape platform. Although the degree to which navigation in this task is guided by spatial information is not yet clear, our findings underscore the fact that the knowledge system subserving place learning can acquire and store place information independently of the actions that the animal must use to navigate to its goal.

\section{REFERENCES}

MorRIs, R. G. M. (1981). Spatial localization does not require the presence of local cues. Leaming \& Motivation, 12, 239-260.

O'KeEFE, J., \& NADEL, L. (1978). The hippocampus as a cognitive map. Oxford: Oxford University Press.

Sutherland, R. J., Chew, G. L., Baker, J. C., \& Linggard, R. C. (1987). Some limitations on the use of distal cues in place navigation by rats. Psychobiology, 15, 48-57.

SUTherLand, R. J., \& LingGard, R. (1982). Being there: A novel demonstration of latent spatial leaming in the rat. Behavioral \& Neural Biology, 36, 103-107.

Tolman, E. C. (1948). Cognitive maps in rats and men. Psychological Review, 55, 189-209.

(Manuscript received October 29, 1987; revision accepted for publication March 21, 1988.) 